\title{
Efficacy of pulsed electromagnetic field therapy in healing of pressure ulcers: A randomized control trial
}

\author{
Anupam Gupta, Arun B. Taly ${ }^{1}$, Abhishek Srivastava, Sendhil Kumar, Murali Thyloth \\ Department of Psychiatric \& Neurological Rebabilitation, ${ }^{1}$ Neurology, National Institute of Mental Health $\&$ Neuro Sciences \\ (NIMHANS), Bangalore, India
}

\author{
Address for correspondence: \\ Dr. Anupam Gupta, \\ Department of Psychiatric \& \\ Neurological Rehabilitation, \\ National Institute of Mental Health \\ $\&$ Neuro Sciences (NIMHANS) \\ Bangalore - 560 029, \\ Karnataka, India. \\ E-mail: anupam@nimhans.kar.nic.in
}

PMID: 19934563

DOI: $10.4103 / 0028-3886.57820$

\begin{abstract}
Background: Pressure ulcers are one of the most common complications in health care settings. Still there are no optimal protocols to manage the pressure ulcers. Aim: To assess the effectiveness of pulsed electromagnetic field therapy (PEMF) in healing of pressure ulcers in patients with neurological disorders. Design: Randomized double blind control trial. Setting: Neurological rehabilitation department in a university research hospital. Participants: Twelve patients (M:F, 9:3) having neurological disorders, with age between 12-50 years (mean 30.16 11.32 yrs) and 24 pressure ulcers. Intervention: Six patients with 13 ulcers received PEMF therapy and the remaining 6 patients with 11 ulcers received sham treatment, for 30 sessions ( 45 minutes each) using the equipment 'Pulsatron'. The frequency of PEMF was set at $1 \mathrm{~Hz}$ with sine waves and current intensity of 30 mili ampere. Whole body exposure was given in both the groups. Outcome Measures: BatesJensen wound assessment tool (BIWAT) score was used as main outcome measure and scores at the end of session were compared with initial scores and analyzed. Similarly National Pressure Ulcer Advisory Panel (NPUAP) scores were compared and analyzed as secondary outcome measure. Results: Thirteen ulcers were in stage IV and II were in stage III at the start of the study. Significant healing of ulcers was noted, BJWAT scores, in both the treatment and sham groups $(P<0.001$ and 0.003 respectively) at the completion of the study. However, when comparing between the groups, healing was not significant $(P=0.361)$. Similarly trend was noted with NPUAP scores with no significant difference between the treatment and sham groups $(P=0.649)$ at the completion of study. Conclusions: No significant difference in pressure ulcer healing was observed between PEMF treatment and sham group in this study.
\end{abstract}

Key words: Neurological illness, pulsed electromagnetic field, pressure ulcers

\section{Introduction}

Pressure ulcers are one of the most common complications in health care settings and the reported incidence varied between 8.5 and $23.9 \%{ }^{[1,2]}$ and the prevalence between 4 and $49 \%,,^{[3-9]}$ depending on the clinical setting. Stage I ulcers are the most common accounting for $48 \%$ of the overall prevalence. ${ }^{[3]}$ The most common sites are sacrum and heel. ${ }^{[3,6,8]}$ A part from risk factors in the Braden's scale, overweight and extreme ages are also significant factors in acute care setting. ${ }^{[1,3]}$
Common physical modalities used to manage pressure ulcers reduce soft tissue inflammation, improve circulation, increase soft tissue extensibility and remodel the scar tissue. ${ }^{[10]}$ There has been increasing interest in the electromagnetic field in the management of ischemic and venous ulcers. It is hypothesized that electrical stimulation influences the migratory, proliferative and synthetic functions of fibroblasts, and also results in increased expression of growth factors. ${ }^{[11]}$ Sheffet et al., ${ }^{[12]}$ suggested the use of moist wound dressing and electrotherapy as adjuvant therapy in the management 
of stage III, IV, and recalcitrant stage II ulcers. Pulsed electromagnetic field (PEMF) therapy is a distinct from electrotherapy. It induces a field effect, not a direct electric effect on the ulcer. ${ }^{[13]}$ PEMF are usually low frequency fields with very specific shape and amplitude and do not exhibit the complications of contact of electrodes. Further, they can be applied in the presence of a cast or wound dressing and the risk of infection is significantly low. ${ }^{[14-16]}$ Therapeutic efficacy of PEMF therapy depends on a number of factors, such as age, general health and gender as well as on the stage of pathology/disease. ${ }^{[14,15]}$ PEMF therapy has been tried in the treatment of stage II and III pressure ulcers in two trials. ${ }^{[17,18]}$ A systematic review has suggested the need for more trails in this area. ${ }^{[19]}$ This randomized control study assessed the efficacy of PEMF therapy stage III or stage IV pressure ulcers in patients with neurological diseases.

\section{Materials and Methods}

This randomized double blind control trial was carried out over a period of six months (July 2006 to December 2006) and included 12 inpatients with neurological disorders having one or more stage III or IV clean and non-infected ulcers (NPUAP, 1989). ${ }^{[20]}$ Pressure ulcers with escher, slough, or infection were debrided, and adequately treated with local and/or systemic antibiotics before recruitment in the study. Patients with cardiac pacemakers and pregnant women were excluded from the study. Nonischemic ulcers and ulcers with underlying osteomyelitis were also excluded from the study. The study had the approval of the Institutional Ethics Committee. After obtaining informed consent, patients were randomly assigned into Group I (treatment group) and Group II (sham group). The sequence for random assignment of the patients was generated by table of random numbers. Six patients (13 ulcers) in group I received PEMF therapy and six patients (11 ulcers) in group II received sham therapy. The end points of the study were: ulcer healing, or completion of 30 sessions after entry into the study, whichever was early. Bates-Jensen wound assessment tool (BJWAT) score was used as main outcome measure and scores at the end of session were compared with initial scores and analyzed. Similarly National Pressure Ulcer Advisory Panel (NPUAP) scores were compared and analyzed as secondary outcome measure

Both groups were given standard pressure ulcer care, practiced in the center. Pressure ulcers in both the groups received daily dressing with normal saline from day one of the study. Patients were blinded about their group assignment (PEMF or sham therapy). All patients were made to lay on a couch, which is a part of the 'Pulsatron' equipment (Madras Institute of
Magnetobiology, Chennai, India) [Figure 1 and Table 1]. The couch is encircled by a metallic frame. Homogenous pulsating electromagnetic field is generated in the frame, covering the whole space within the frame (including the patients). The equipment provides a low intensity and ultra low frequency homogenous magnetic field. The patient lies comfortably in supine or prone position. Patients in Group I (treatment group) received $1 \mathrm{~Hz}$ frequency sine waves with 30 mili ampere current intensity. Dressing over the pressure ulcers were not removed during the therapy sessions. Patients were exposed to PEMF for 30 sessions, each of 45-min duration. Therapy was given for five days a week till the end points of the study. For patients in Group II (the sham group) similar protocol was followed but PEMF equipment was not switched-on.

The wound healing was assessed by Bates Jensen Wound Assessment Tool (BJWAT) ${ }^{[21]}$ and ulcer staging by National Pressure Ulcer Advisory Panel (NPUAP) protocol. ${ }^{[20]}$ BJWAT gives pressure ulcers a score from 13 to 60 with lower score indicating a better status or regenerating wound and higher score indicating wound deterioration or degeneration on a wound status continuum. There are four ulcer stages in NPUAP staging: stage I: Defined area of persistent redness or persistent red, blue or purple hue; stage II: Partial thickness skin loss involving epidermis and/or

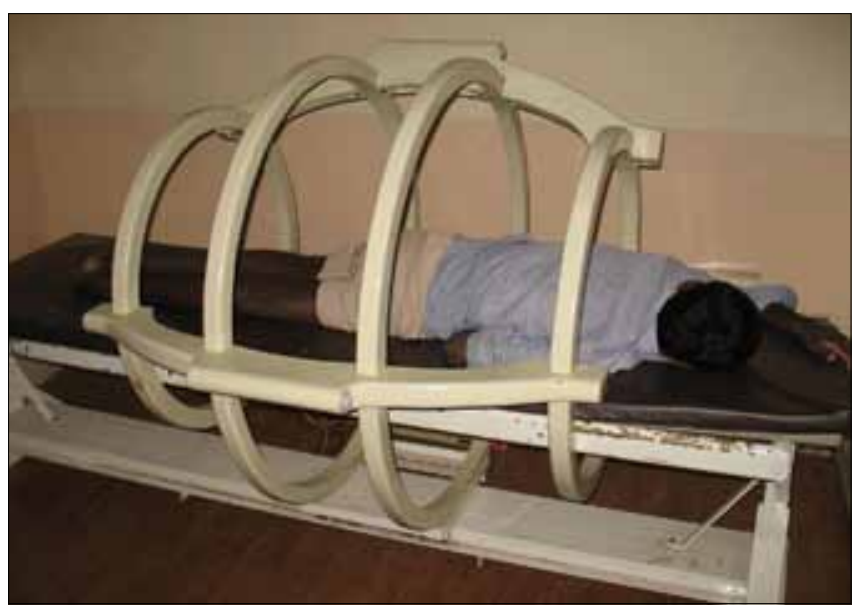

Figure 1: Pulsed magnetic field therapy equipment

Table 1: Specifications of equipment 'Pulsatron'

\begin{tabular}{ll}
\hline Features & Specifications \\
\hline Weight & $40 \mathrm{~kg}$ \\
Dimensions $(\mathrm{L} \times \mathrm{B} \times \mathrm{H})$ & $7 \mathrm{ft} \times 4 \mathrm{ft} \times 4 \mathrm{ft}$ \\
Field intensity & $750-1500$ nano Tesla \\
Accuracy & $+/-50$ nano Tesla \\
Wave forms & Square and sine \\
Frequency range & $0-100 \mathrm{~Hz}$ \\
DC fields & Can be delivered \\
Power & $22 \mathrm{~V} \mathrm{AC}, 50-60 \mathrm{~Hz}$ \\
Power consumption & 10 watts (Maximum) \\
\hline
\end{tabular}


dermis; stage III: Full thickness skin loss with damage and necrosis to subcutaneous tissue with wound extending down but not through underlying fascia; and stage IV: Full thickness skin loss with tissue destruction, damage to muscle, tendon, bone or joint capsule. Colored photographs of ulcers were taken at the time of recruitment into the study and at the end treatment protocol. An investigator/evaluator, blind to the randomization of the ulcers, assessed the pressure sores weekly and recorded the observations using BJWAT and NPUAP ulcer stage form.

\section{Statistical analysis}

Data were analyzed using SPSS version 11.0 software. Data were described using frequencies; mean, median, standard deviation, minimum, and maximum values. Comparison between treatment and sham group was done with Mann-Whitney U test for BJWAT and NPUAP ulcer grade, whereas Wilcoxon signed rank test was used to compare within the group. The two-tailed level of significance was kept at 0.05 . Intention to treat analysis was used.

\section{Results}

The study included 12 patients (M:F, 9:3), six (mean age $27.83 \pm 14.64$ yrs, (12-50 yrs) in Group I (treatment group) and six (mean age $28.83 \pm 11.10$ yrs (15-48 yrs) in Group II (sham group). The mean duration of the illness at the beginning of study was $6.42 \pm 6.40$ months (one to 20 months) while the mean duration of pressure ulcer was $103.75 \pm 113.70$ days (10 to 420 days). The mean duration of stay in the rehabilitation unit was $98.66 \pm 66.58$ days (24-193 days). The number of treatment sessions in patients ranged from 22-30, mean of $29.06 \pm 2.29$. There were no non-compliers or dropouts from the study.

Patients' demographic profile, diagnosis, site of pressure ulcer, at-admission and at-discharge BJWAT and NPUAP ulcer staging is given in Table 2 . The most common site of pressure ulcer was sacral region $(\mathrm{n}=9)$, followed by gluteal region $(\mathrm{n}=6)$, heel $(\mathrm{n}=5)$. Thirteen ulcers $(54.16 \%)$ were stage IV (eight and five in treatment and sham groups, respectively) and 11 ulcers $(45.84 \%)$ were stage III (five and six in treatment and sham groups, respectively) at the beginning of the study. At the end of the study, two patients had stage I ulcers $(8.33 \%)$, four had stage II (16.67\%), 16 had stage III (66.67\%), and two had stage IV ulcers $(8.33 \%)$.

There was significant healing of the ulcers (NPUAP ulcer stage) at the end of the study in both the treatment and sham groups $(P=0.008$ and 0.014 , respectively). However, when comparision was done between treatment goup and sham group there was no significant difference $(P=0.649)$. There was significant differences in BJWAT scores, admission and discharge, in both treatment and sham group $(P=0.001$ and 0.003 , respectively, $)$. However, when the comparisons were made between the groups there mas no significant difference $(P=0.361)$. Two patients $(8.33 \%)$ showed complete healing of pressure ulcers in less than 30 sessions and both of thme received PEMF therapy. In none of the patients there was worsening of pressure ulcers (both groups) and there were no complications attributable to pulsed electromagnetic field (PEMF) therapy.

\section{Discussion}

Several studies have assessed the efficacy of PEMF therapy in the healing of pressure ulcers. Two of them were randomized control trials ${ }^{[17,18]}$ and others are prospective studies. ${ }^{[12,22]}$ The efficacy of PEMF has also been studied in patients with venous ulcers ${ }^{[13,23,24]}$ and in leprosy patients with nonhealing ulcers. ${ }^{[25]}$ In the randomized control trail by Comorosan et al., ${ }^{[17]} 20$ patients received PEMF and five patients each received conventional therapy and conventional therapy and sham therapy for a maximum period of two weeks. Healing of pressure ulcers was significant in the treatment group compared to other two groups. ${ }^{[17]}$ In the other randomized control trial by Salzburg et al., (1995), ${ }^{[18]} 30$ male patients with spinal cord injury and grade II and III pressure ulcers were given either PEMF or sham treatment for 12 weeks or until the ulcers healed. Authors noted no significant difference between the treatment and the sham group in healing of pressure ulcers. Healing was better in stage II ulcers in the treatment group. Systemic review of the efficacy of PEMF in the healing of pressure ulcers by Olyaee et al. ${ }^{[19]}$ concluded that the present data provide no reliable evidence of benefit from electromagnetic field therapy and recommended more randomized control trials with larger sample size.

In the present randomized control trial study, BJWAT scores and NPUAP ulcer staging were used for assessment of healing of pressure ulcers. No significant difference in the ulcer healing was found between treatment and sham group using both the scales. However the sample size was too small. Other measures, such as, supportive surfaces, turning and bed positioning, moist dressing, debridement of the Escher, and adequate nutrition have important contribution in healing of ulcers. How much each of these measures have contributed for healing of pressure ulcers, was difficult to ascertain in this study because of the small sample size and inability to exclude confounding effects of these measures provided to both the groups.

Pressure ulcers in stage III and IV usually require surgical closure and probably electromagnetic therapy 


\begin{tabular}{|c|c|c|c|c|c|c|c|c|}
\hline $\begin{array}{l}\text { Age } \\
\text { (Yrs) }\end{array}$ & Sex & Diagnosis & $\begin{array}{l}\text { Tt.vs } \\
\text { Sham }\end{array}$ & $\begin{array}{l}\text { Site of } \\
\text { ulcer }\end{array}$ & $\begin{array}{l}\text { BJWAT } \\
\text { score (I) }\end{array}$ & $\begin{array}{l}\text { BJWAT score } \\
\text { (Compl.) }\end{array}$ & $\begin{array}{l}\text { Ul.grade } \\
\text { (I) }\end{array}$ & $\begin{array}{l}\text { Ul.grade } \\
\text { (Compl.) }\end{array}$ \\
\hline \multirow[t]{2}{*}{50} & \multirow[t]{2}{*}{ M } & \multirow[t]{2}{*}{ Pott's spine } & \multirow[t]{2}{*}{ Tt. } & Sacral & 42 & 23 & 4 & 3 \\
\hline & & & & Lt.GT & 37 & 22 & 4 & 3 \\
\hline \multirow[t]{2}{*}{15} & \multirow[t]{2}{*}{ M } & \multirow[t]{2}{*}{ Pott's spine } & \multirow[t]{2}{*}{ Sham } & Sacral & 43 & 22 & 4 & 3 \\
\hline & & & & Lt.Glut & 27 & 18 & 4 & 4 \\
\hline 36 & $\mathrm{~F}$ & TBM & Sham & Sacral & 26 & 17 & 4 & 3 \\
\hline \multirow{2}{*}{12} & \multirow{2}{*}{ M } & \multirow{2}{*}{ ADEM } & \multirow{2}{*}{ Tt. } & Rt. heel & 43 & 25 & 4 & 4 \\
\hline & & & & Lt.Glut & 24 & 13 & 3 & 1 \\
\hline \multirow[t]{3}{*}{34} & \multirow[t]{3}{*}{$\mathrm{F}$} & \multirow[t]{3}{*}{ Traumatic myelopathy } & \multirow[t]{3}{*}{ Sham } & Sacral & 32 & 21 & 3 & 2 \\
\hline & & & & Lt. heel & 37 & 20 & 3 & 2 \\
\hline & & & & Lt.Glut & 36 & 29 & 4 & 3 \\
\hline 20 & M & D-L Epend & Tt. & Sacral & 37 & 21 & 4 & 3 \\
\hline 30 & M & TBI with sequelae & Sham & Rt.Glut & 29 & 23 & 4 & 3 \\
\hline \multirow[t]{3}{*}{27} & \multirow[t]{3}{*}{ M } & \multirow[t]{3}{*}{ Traumatic myelopathy } & \multirow[t]{3}{*}{ Tt. } & Rt. Heel & 45 & 16 & 4 & 3 \\
\hline & & & & Rt.GT & 34 & 21 & 3 & 3 \\
\hline & & & & Lt.Glut & 39 & 24 & 3 & 2 \\
\hline \multirow[t]{2}{*}{30} & \multirow[t]{2}{*}{$\mathrm{F}$} & \multirow[t]{2}{*}{ Traumatic myelopathy } & \multirow[t]{2}{*}{ Tt. } & Sacral & 26 & 17 & 4 & 3 \\
\hline & & & & Rt.GT & 22 & 19 & 4 & 3 \\
\hline \multirow[t]{2}{*}{48} & \multirow[t]{2}{*}{ M } & \multirow[t]{2}{*}{ Traumatic myelopathy } & \multirow[t]{2}{*}{ Sham } & Sacral & 31 & 18 & 3 & 3 \\
\hline & & & & Lt. heel & 25 & 20 & 3 & 3 \\
\hline \multirow[t]{3}{*}{14} & \multirow[t]{3}{*}{ M } & \multirow[t]{3}{*}{ Cervical florosis } & \multirow[t]{3}{*}{ Tt. } & Sacral & 32 & 25 & 4 & 3 \\
\hline & & & & Rt. heel & 29 & 13 & 3 & 1 \\
\hline & & & & Lt.Glut & 33 & 22 & 3 & 3 \\
\hline 24 & M & Traumatic myelopathy & Sham & Sacral & 25 & 20 & 3 & 2 \\
\hline & & & & Rt.GT & 22 & 19 & 3 & 3 \\
\hline
\end{tabular}

Tt - Treatment; BJWAT - Bates Jensen Wound Assessment Tool; I - Initial scores; Compl. - Completion of study; UI. Grade - Ulcer grade according to NPUAP; Lt. GT-Left Great Trochanter; Rt. GT - Right Great Trochanter; Lt. Glut. - Left Gluteal region; Rt. Glut. - Right Gluteal region; TBM - Tubercular Meningitis; ADEM - Acute Demyelinating Encephalo-myelitis; D-L Epend. - Dorso-lumber Ependymoma

as an adjuvant therapy. Earlier randomized control studies $^{[17,18]}$ included stage II and III ulcers in their study. In the present study, no deliberate attempt was made to include stage IV ulcers and the patients admitted during the study period had only stage III and IV pressure ulcers. Four patients underwent surgery (one from treatment group and three from sham group); two patients required skin grafting and two needed flap mobilization and closure of the wound after completion of treatment sessions.

There are certain limitations of the present study, such as, the small sample size, use of restricted frequency of PEMF $(1 \mathrm{~Hz})$, and short duration of exposure. Only stage III and IV ulcers were included in the study. Ulcers in these stages usually require surgical closure and electromagnetic therapy has to be used as an adjuvant therapy. Only $1 \mathrm{~Hz}$ frequency was used as advised by the manufacturer. Same frequency was used in the earlier studies using the same equipment for the treatment of pressure ulcers. Probably feature randomized trails should consider the frequency and period of exposure of PEMF, stage of ulcer, large sample sized while designing the study.

\section{References}

1. Fife C, Otto G, Capsuto EG, Brandt K, Lyssy K, Murphy K, et al. Incidence of pressure ulcers in a neurologic intensive care unit. Crit Care Med 2001;29:283-90.
2. Bergstrom N, Braden B, Kemp M, Champagne M, Ruby E. Multi-site study of incidence of pressure ulcers and the relationship between risk level, demographic characteristics, diagnoses and prescription of preventive interventions. J Am Geriatr Soc 1996;44:22-30.

3. Groeneveld A, Anderson M, Allen S, Bressmer S, Golberg M, Magee B, et al. The prevalence of pressure ulcers in a tertiary care pediatric and adult hospital. J Wound Ostomy Continence Nurs 2004;31:121-2.

4. Gunningberg L. EPUAP pressure ulcer prevalence survey in Sweden: A two-year follow-up of quality indicators. J Wound Ostomy Continence Nurs 2006;33:258-66.

5. Shahin ES, Dassen T, Halfens RJ. Pressure ulcer prevalence and incidence in intensive care patients: A literature review. Nurs Crit Care 2008;13:71-9.

6. Lahmann NA, Halfens RJ, Dassen T. Pressure ulcers in German nursing homes and acute care hospitals: Prevalence, frequency and ulcer characteristies. Ostomy Wound Manage 2006;52:20-33.

7. Capon A, Pavoni N, Mastromattei A, Di Lallo D. Pressure lucer risk in longterm units: Prevalence and associated factors. J Adv Nurs 2007;58:263-72.

8. Vanderwee K, Clark M, Dealey C, Gunningberg L, Defloor T. Pressure ulcer prevalence in Europe: A pilot study. J Eval Clin Pract 2007;13:227-35

9. Uzun O, Tan M. A prospective, descriptive pressure ulcer risk factor and prevalence study at a university hospital in turkey. Ostomy Wound Manage 2007;53:44-56.

10. Sussman C. Management of wound healing with physical therapy technologies. In: Sussmann C and Bates- Jansen BM (editors) Wound care A collaborative practice manual for physical therapists and nurses. Aspen publishers: Maryland;1998. p. 347-56.

11. Weiss DS, Kirsner R, Eaglstein WH. Electrical stimulation and wound healing. Arch Dermatol 1990;126:222-5.

12. Sheffet A, Cytryn AS, Louria DB. Applying electric and electromagnetic energy as adjuvant treatment for pressure ulcers: A critical review. Ostomy Wound Manage 2000;46:28-33,36-40,42-4.

13. Stiller MJ, Pak GH, Shupack JL, Thaler S, Kenny C, Jondreau L. A portable pulsed electromagnetic field (PEMF) device to enhance healing of recalcitrant venous ulcers: A double-blind, placebo-con-trolled clinical trial. Br J Dermatol 1992;127:147-54. 
14. Markov MS. How to go for magnetic field therapy. EMF therapeutics Inc.; 5-15.

15. Markov MS, Todorov NG. Electromagnetic field stimulation of some physiological processes. Studia Biophysica 1984;99-151-6.

16. Markov MS. Magnetic and electromagnetic fields-a new frontier in clinical biology and medicine. Proceedings of the Millennium International Workshop on Biological Effects in Biology and MedicineCrete 17- 20 October 2000363-372 ISBN 960-86733-0-5.

17. Comorosan S, Vasilco R, Arghiropol M, Paslaru L, Jieanu V, Stelea S. The effect of Diapulse therapy on the healing of decubitus ulcer. Rom J Physiol 1993;30:41-5.

18. Salzberg CA, Cooper-Vastola SA, Perez F, Viehbeck MG, Byrne DW. The effects of nonthermal pulsed electromagnetic energy on wound healing of pressure ulcers in spinal cord-injured patients: A randomized, doubleblind study. Ostomy Wound Manage 1995;41:42-446, 48 passim.

19. Olyaee MA, Flemming K, Cullum NA, Ravaghi H. Electromagnetic therapy for treating pressure ulcers. Cochrane Database of Systematic Reviews. 3.2006.

20. National Pressure Ulcer Advisory Panel. Consensus development conference statement. 1989.
21. Bates Jansen BM. Tools to measure wound healing. In Sussmann C, Bates-Jansen BM. Wound care: A collaborative practice manual for physical therapists and nurses. Aspn publishers: Maryland; 1998. p. 115-6.

22. Itoh M, Montemayor JS Jr, Matsumoto E, Eason A, Lee MH, Folk FS Accelerated wound healing of pressure ulcers by pulsed high peak power ecectrmagnetic energy (Diapulse). Decubitus 1991;4:24-529'-34.

23. Ieran M, Zaffuto S, Bagnacani M, Annovi M, Moratti A, Cadossi R. Effect of low frequency pulsing electromagnetic fields on skin ulcer of veous origin in humans: A double-blind study. J Orthop Res 1990;8:276-82.

24. Kenkre JE, Hobbs FD, Carter YH, Holder RL, Holmes EP. A randomized controlled trial of electromagnetic therapy in the primary care management of venous leg ulceration. Fam Pract 1996;13:236-41.

25. Sarma GR, Subrahmanyam S, Deenabandhu A, Babu CR, Madhivathanan S, Kesavaraj N. Exposure to pulsed magnetic fields in the treatment of plantar ulcers in leprosy patients-A pilot, randomized, double-blind, controlled clinical trial. Indian J Lepr 1997;69:241-50.

Accepted on 06-03-2009

Source of Support: Nil, Conflict of Interest: None declared. 